NASA's Einstein Observatory has been extensively used. Through the unprecedented powers of this $X$-ray telescope, several new $X$-ray sources have been discovered in connection with UGO's. However no firm, one-to-one association can be claimed as yet. Here again, work is still in progress, with special emphasis on the 2CG $195+4$ source, discovered by SAS-2 and dubbed "Geminga" by the COS-B workers, some of whom are now pondering on the preliminary intriguing $X$-ray data of the Geminga enigma.

\section{Future Prospects for Gamma-Ray As- tronomy}

With the last of the $\gamma$-ray telescopes just passed away, it is natural to enquire on the future steps to take for advancing highenergy astrophysics. Theoretically, there is a serious need for a better understanding of the UGO's as well as of the emission from pulsars and AGNs; the problem here would seem to be the absence of discrimination between the large variety of models proposed, or alternatively, with the lack of one sufficiently accurate model. Observations at other wavelengths are crucial, but at the same time not easy. The understanding of the diffuse emission from the ISM and of that from local structures and molecular clouds seems to be pinned down reasonably well at the moment, to the point that this aspect of $\gamma$-ray astronomy is now considered a new tool for the tracing of the diffuse galactic matter.

Besides better theoretical work however, clearly better instuments are also needed, and the direction to go is that of improving the angular resolution, definitely the main current $\gamma$-ray astronomy limitation. A lot can be achieved by designing better spark chambers of the "classic" type so as to minimize the basic physics effects responsible for the angular uncertainty and at the same time decrease the background. An instrument of this type, also characterized by a very large sensitive area, is being planned for the NASA Gamma-Ray Observatory by a U.S.-German collaboration, and will fly before the end of the eighties. A different approach is used by the Soviet-FrancoItalian mission 'Gamma 1', to be flown hopefully in less than two years time. It uses a "random" mask collimation technique, aiready applied succesfully in other branches of astronomy as well as in ground-based, non-astronomical equipment. An angular resolution of $-1-5$ arc min on a few bright sources could be obtained, thus tremendously reducing the sizes of the UGO error boxes and the corresponding number of candidate counterparts. Because of its nature, this mission can be considered as complementary to GRO, and eventually from the joint data base it should be possible to make a solid step forward in this energetic window on the Universe.

\title{
PERMANENT MAGNETS
}

\section{Characterization and Materials Development}

\author{
Ch. Gnehm, Lupfig, $\mathrm{CH}$ \\ (Ugimag Recoma $A G$ )
}

The ferromagnetic materials, which are characterized by their very high magnetic permeability $\mu$, may be clearly divided into two groups: the magnetically soft (easy to magnetize and demagnetize) and the magnetically hard (hard to magnetize and demagnetize). Even in the absence of an initially applied external magnetic field, both groups of material posses their own magnetic field. In both, the ease of magnetization and therefore the behaviour under load, is determined by the structure of the magnetic domains whereas the magnitude of the saturation magnetization is not structure-sensitive, but depends on the phase of the material.

In soft magnetic materials, the boundaries of the magnetic domains need to move as freely as possible which is achieved by avoiding trapping by impurities, crystal or grain boundaries, strain centres etc. The job of this group of materials is chiefly the multiplying of magnetic flux in electromagnetic machines and devices. The other group, the magnetically hard materials, find their use as bearers for magnetic recordings or as permanent ma gnets. After the initial magnetization, a permanent magnet must be able to resist the effects of demagnetizing fields, including its own, so that high stability of the magnetic domains is a primary requirement. In other words, domain walls should not move and the polarity of the domains should remain in the original state.

\section{Working Principle of Permanent Ma- gnets}

In the following, we shall confine our attention to the permanent magnets. They are of great importance as industrial products and find their application in a wide variety of electro-mechanical transducer systems. These are typically high performance electric motors, generators, positioning and holding devices, instruments, microwave and focussing tubes, loudspeakers, bearings, clutches; we find permanent magnets in household products, consumer goods, light and heavy industrial machinery, car equipment, precision instruments, telecommunications, data processing, aircraft and space equipment.

In all these practical cases, the magnet itself is fundamentally an energy-storage device which does no net work on its surroundings. Its only function is to provide an external magnetic field. Therefore, it must have free poles. In other words, a magnet always operates on open circuit. The efficiency of a permanent magnet system is very sensitive to the demagnetization curve of the permanent magnet material under consideration, more specifically to the intersection of the load line OP $10=$ origin, $\mathrm{P}=$ operating point) with this curve. Fig. 1 shows the demagnetization curve as the second quadrant part of the hysteresis loop of a permanent magnet in an external ma gnetic field $H$. The slope of the load line OP is equal to $-\left(4 \pi-N_{d}\right) / N_{d}$ where $N_{d}$ (demagnetizing factor) is defined by the length / diameter ratio of the magnet in a fixed position.

The demagnetizing field $H_{\mathrm{d}}=N_{\mathrm{d}} \times M$ ( $M=$ magnetization) is one of the most fundamental parameters in any open permanent magnet circuit systern. This is owing to the fact that the permeability $\mu$ defined as the extent to which an originally unmagnetized body disturbs a magnetic field into which it is placed - is always the result of an interaction between the outside field or the field in the air gap and the field created inside the magnet.

The designer of a permanent magnet system usually attempts to make the best use of the available materials by choosing operating points with maximum product $B \times H$ i.e. $(B H)_{\max }$ but as many applications deal not only with static open circuits, but also with dynamic conditions, the internal self-demagnetizing field will not always remain constant and so the slope of the load line will change. In this case, the demagnetization curve should approximate a straight line with maximum coercive field. In practice, however, the choice of material and design is more complicated by other requirements such as mechanical, thermal and environmental stability, optimum weight and volume, and of course by material and manufacturing costs, which in turn may vary considerably as a function of ductility or machinability and other physical properties.

\section{Historical Development of Materials}

The history of permanent magnets starts with the compass needle, which was already in use in early Chinese culture. The first synthetic permanent magnets were obtained by rubbing a piece of iron with the naturally occurring magnetite $\mathrm{Fe}_{3} \mathrm{O}_{4}$, also called lodestone. In the early $19^{\text {th }}$ century, the experiments and theories of Oersted, Ampère, Faraday and their contemporaries resulted in the construction of the first 


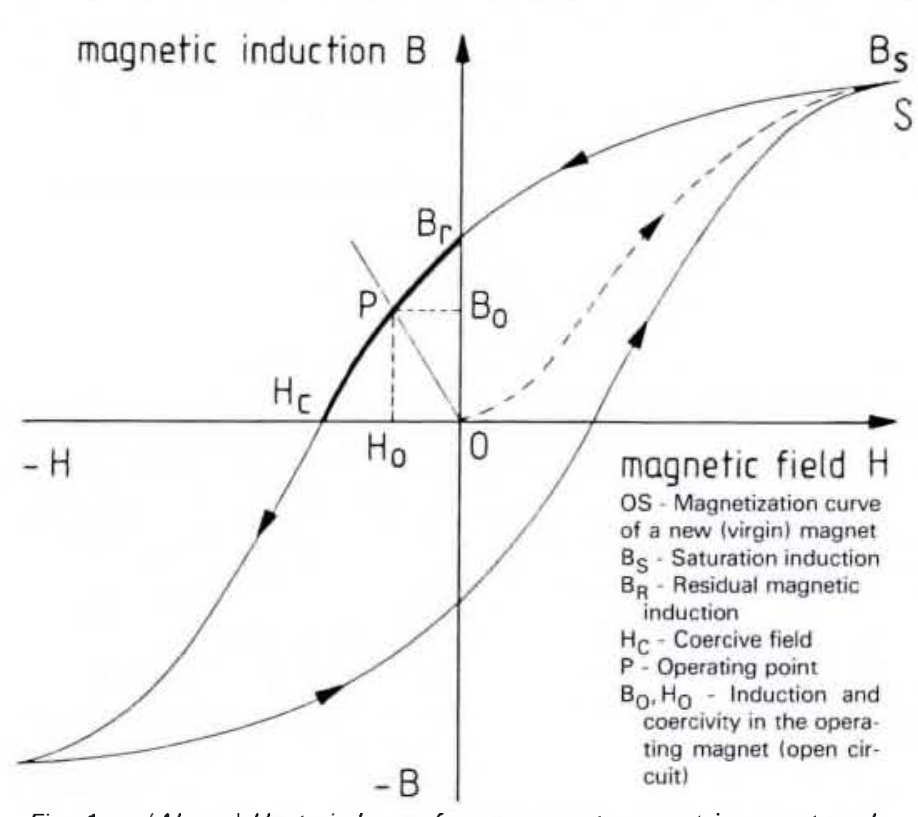

Fig. 1 - (Above) Hysteris loop of a permanent magnet in an external magnetic field $H$.

Fig. 2 - (Right) Typical demagnetization curves of ferrites, alnicos and rare earth-cobalt permanent magnets.

electromagnetic machinery which made use of magnetic induction. U-shaped steel pieces, magnetized in electromagnetic fields, were soon known to lift more than ten times their own weight and martensic hardened steels then became accepted as the best material for permanent magnets in technical applications, until the 1930's. It is only in the past 50 years that a remarkable advent of new material types has occurred. Available coercivities have increased over this period from about 250 Oe by more than 100-fold, and the increase of the energy product has been more than 25-fold.

The metallic alloys which started to open new horizons for permanent magnet applications before World War II were of the types $\mathrm{Al}-\mathrm{Ni}, \mathrm{Mn}-\mathrm{Ag}-\mathrm{Al}, \mathrm{Fe}-\mathrm{Co}-\mathrm{Mo}$ (Remalloy), Pt-Co, Cu-Ni-Co, Cu-Ni-Fe, Fe-Co-U (Vicalloy) and, most important, Al-Ni-Co (Alnico). In the 1950's, the ferrites took us a further very important step in magnet technology. As oxides having the chemical formula $\mathrm{BaO} \times 6 \mathrm{Fe}_{2} \mathrm{O}_{3}$ or $\mathrm{SrO} \times 6 \mathrm{Fe}_{2} \mathrm{O}_{3}$, they are mechanically hard and brittle, and because their production process is very similar to that of ceramics, both barium and strontium ferrites are often called ceramic magnets.

Other modern materials with remarkable permanent magnetic properties are the $\mathrm{Fe}$ $\mathrm{Cr}$-Co and Mn-Al type alloys. Since 1968, the rare earth-cobalt permanent magnets have found a place in many new industrial applications for which they must be considered a key material.

This enumeration of well known permanent magnet materials is of course not complete. Fig. 2 shows the typical demagnetization curves of today's three families of most widely used materials, namely:
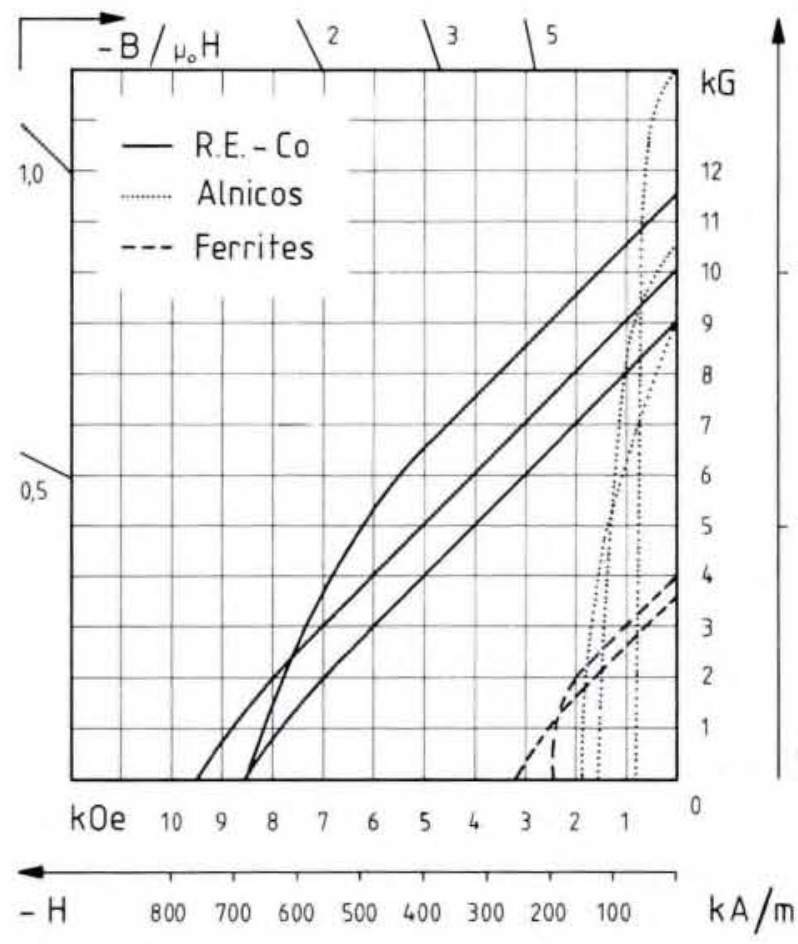

- the ferrites with satisfactory coercivity but relatively low remanence,

- the alnicos with very high remanence but generally low coercivity.

the rare earth-cobalt magnets with maximum energy product $(B H)_{\text {max }}$

Owving to their different characteristics in performance and cost - ferrites are the cheapest and rare earth-cobalt the most expensive - it is evident that their markets and applications are complementary. As can be seen from Fig. 2, each family consists of several types which form a "palette" from which can be chosen the most suitable for the particular technical application. The world market of permanent magnets represents actually a value of approx. $600 \mathrm{M} \$$, ferrites and alnicos taking about 50 and $40 \%$, respectively.

\section{Making Performant Magnets}

Permanent magnets consist of ferromagnetic particles in a polycrystalline material. In the ferrites and rare earth-cobalt magnets, these particles are produced by direct comminution of bulk material and the further processing is mechanical compaction and densifying at high temperature as in ceramics or powder metallurgy. In the alnicos, the fine-particle structure is the result of a metallurgical precipitation reaction that takes place in the solid state. Alnicos are obtained by casting or sintering of polycrystalline grains of the alloy.

All high-performance permanent magnets are anisotropic, because their particles have been oriented during the manufacturing process, so that even macroscopically, they have an axis of easy magnetization. There are just a few exceptions to this to cover the demand for isotropic magnets as when one piece of magnet is designed to be magnetized in several direc- tions. In this case, the overall performance in terms of the energy product $(B H)_{\max }$ is considerably lower than in an anisotropic magnet of the same composition.

The saturation magnetic induction $B_{\mathrm{s}}$ is an intrinsic property of a given material. It defines the theoretical limit to the maximum energy product $(B H)_{\max }$ which cannot exceed $B_{s}^{2} / 4$. This limit is approached by a perfect alignment of the magnetic easy axes of the particles, so that the ratio of remanence to saturation $B / B_{\mathrm{s}}$ is close to unity. But the primary magnitude of a permanent magnet is its hardness or coercivity, whose mechanisms are more complex and difficult to understand, especially when dealing with the statistics of imperfections in the microstructure of polycrystalline material. Magnetization reversal in a permanent magnet occurs in two ways, either by nucleation and growth of reverse domains, and/or by coherent or incoherent rotation of the magnetization direction within a magnetic domain.

The instruction how to make a performant permanent magnet may thus be summarized as follows: find a material with the highest saturation induction, align it perfectly, prepare it in a dense form and prevent magnetization reversal. Furthermore, as it is known that the atomic spin directions become randomly oriented above a defined temperature $T$ (Curie-temperature), it is obvious that $T$ for this material must at least be higher than the ambient or working temperature.

To the magnet materials scientist, the most challenging part of the task is to understand, control and improve the coercivity. Table 1 shows a very simplified generalization of the hardening mechanisms in permanent magnets, which allows a rough classification of materials. 
In magnet steels, Remalloy and $\mathrm{Mn} \mathrm{Al}$, where magnetization changes by wall motion are hindered only mechanically by precipitates, the strain and crystal defects - similar to the mechanical hardening which occurs at the same time - are of low importance. We shall therefore, look closer at the concepts of fine particles and anisotropy. If the anisotropy refers to the shape of particles or grains, the intrinsic coercivity $H_{\mathrm{ci}}$ is directly proportional to the saturation magnetization $M_{\mathrm{s}}$ and to the difference in the demagnetizing factors in the long and short axis of the particle. This mechanism requires the magnetic phase to be embedded in a non-magnetic matrix, which always means dilution of the magnet. As a result, the still important art of making alnicos consists in finding the optimum phase compositions, perfecting the metallurgical casting methods for preferred orientation of regular grain structures, and applying appropriate thermal annealing, mostly in a magnetic field.

The crystal anisotropy coercive force is, on the other hand defined by $H_{c}=2 K / M_{s}$, where $K$ is a constant describing the magnetocrystalline anisotropy of the material. The most successful approach to finding high-performance permanent magnet material has therefore been to search for substances with the best combination of the primary magnetic properties $M_{\mathrm{s}^{\prime}} K$ and $T$. The well-known outcome is the rare earth-cobalt magnets, where two groups can be distinguished: the $\mathrm{Sm} \mathrm{Co}$ which is basically a single-phase material, and the $\mathrm{Sm}_{2}$ (Co $\left.+\mathrm{X}\right)_{17}$, which is precipitation hardened. $\mathrm{Sm} \mathrm{Co} \mathrm{Co}_{5}$ owes its nearly ideal, extremely high coercive force to the nucleation and/or pinning of domain walls at surfaces or grain boundaries.

A large amount of samarium can be substituted by neodymium, praesodymium or cerium-mischmetal, with the aim of increasing the saturation magnetization $/ \mathrm{Nd}$ and $\mathrm{Pr}$ ) or decreasing raw material cost (mainly with mischmetal). The $\mathrm{Sm}_{2}$ (Co + $X)_{17}$ material offers the absolutely highest values in remanence and energy product which is commercially available today. Its coercive force, which is not yet completely satisfactory but shows a potential for further improvement, is controlled by the pinning of domain walls on coherent precipitates, either of $R_{2} T M_{17}$ phase $(R=$ rare earth, $T M=$ transition metal) embedded in a $R T M_{5}$ matrix or of discrete $R T M_{5}$ particles embedded in $R_{2} T M_{17}$. The metals which are used as partial substitute for cobalt and which - besides the thermal treatment - are essential for this hardening mechanism, are mainly $\mathrm{Cu}, \mathrm{Fe}, \mathrm{V}, \mathrm{Ti}$, $\mathrm{Hf}, \mathrm{Cr}, \mathrm{Zr}, \mathrm{Mn}, \mathrm{Nb}$.

\section{Magnet Stability}

If permanent magnets were to operate in carefully controlled, optimized and constant environmental conditions with respect to temperature, chemicals and external magnetic fields, they could provide exactly the same magnetic flux over an infinite length of time. But in real applications, permanent magnets have some difficulties in properly living up to their name.

If the external magnetic field in an opencircuit permanent magnet system changes to the positive side $(\triangle H$ = positive), the change in induction $B$ is small and the magnet can return back to its original operating point. This reversibility is not guaranteed if $\triangle H$ is negative and if the demagnetization curve of the permanent magnet is not an ideal straight line with slope $B / H=1$. This problem is often overcome in practice by magnetic stabilizing, which is achieved by subjecting a fully magnetized magnet to a demagnetizing field that moves its operating point down a sufficient amount. After this knock-down, which is at the cost of remanent induction, the demagnetization curve approaches the ideal straight line and the magnet better resists demagnetizing fields. In practice, it is well known that the Alnico magnets especially can be demagnetized or - to the great surprise of the person ignorant of these effects - loose their "magnetic power" by simply removing them from their circuit and replacing.

The heating and cooling of permanent magnets normally affect their magnetic properties. We can distinguish three different types of magnetization changes:

(1) Reversible changes of magnetization with temperature; these are directly related to the temperature dependence of the saturation magnetization.

(2) Irreversible changes associated with changes in the magnetic structure; these can be recovered by remagnetization after returning to room temperature.

(3) Irreversible changes related to metallurgical or chemical changes; these cannot be recovered by remagnetization.

The reversible temperature coefficient for the induction is quite small for the alnicos, of the order of -0.01 to -0.02 $\% /$ deg. This coefficient usually depends on the slope of the load line and to a great extent on the temperature range under consideration. For the ferrites, $\triangle B / B$ is approximately $-0.02 \% / \mathrm{deg}$ and $\triangle H_{\mathrm{c}} / H_{\mathrm{c}}$ is approximately $+0.3 \% / \mathrm{deg}$. The rare earth-cobalt magnets show a temperature coefficient $\triangle B / B$, of the order of -0.03 to $-0.05 \% / \mathrm{deg}$; for special cases, however, it is possible to adjust this coefficient to zero or to any intermediate value by adding heavy rare earth elements such as gadolinium. This is explained by the positive temperature coefficient of $\mathrm{Gd} \mathrm{Co}_{5}$.

The irreversible change in magnetic induction caused by temperature changes is understood as an effect of thermal energy which causes a small and slow decrease in magnetization, even at constant temperature, by irreversible rotations or domain

\section{A new and different publication from SPIE- The International Society for Optical Engineering:}

\section{OPTICAL ANECDOTES by D.J. Lovell}

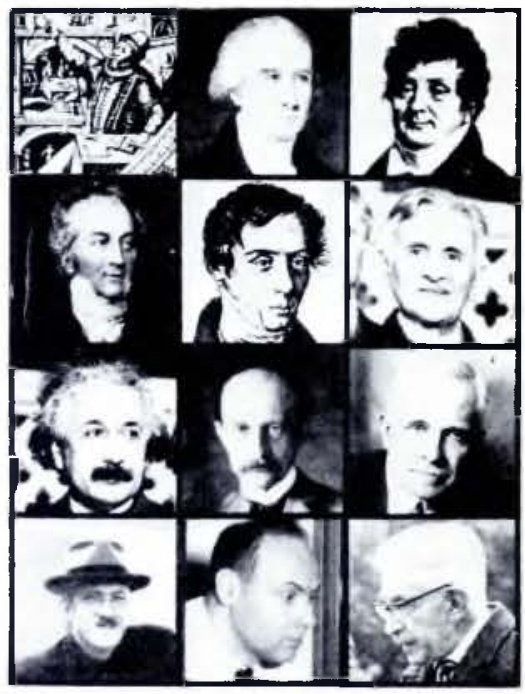

36 delightful stories about the personal lives and adventures of men who have made significant contributions to optics while seeking to understand the nature of light-from 3500 B.C. to A.D. 1981.

$\star$ Inspirational reading for anyone in
optics

$\star$ A useful source book for teachers

$\star$ A stimulating reference for students

* A highly appropriate gift for your optical engineering colleagues, clients, and students

$6 \times 9$ paperback $\bullet 134$ pages $\bullet 27$ illustrations $\bullet$ ISBN 0-89252-325-3

SPIE Members: $\$ 17$ - Nonmembers: $\$ 20$ - Overseas: $\$ 25$. Quantity discounts available.

Order from SPIE, P.O. Box 10, Bellingham WA 98227-0010 USA. Telephone 206/6763290. Orders must be prepaid (in U.S. funds) or accompanied by a purchase order. California residents add $6 \%$ sales tax; Washington State residents, $6.3 \%$. Prices include shipping at book rate. For faster delivery via UPS (U.S. only), add $\$ 1.50$ per book. Street address must be given for UPS delivery. 
wall movements. Because this change becomes smaller for each successive temperature cycle, a permanent magnet can be stabilized against the effect by cycling it repeatedly over a sufficiently wide temperature range. Alnicos which are properly stabilized by thermal cycling and magnetic knock-down produce fluxes which are constant to less than $\pm 5 \times 10^{6}$ over a period of one year.

The long-term stability is no problem with modern permanent magnet materials. In rare earth-cobalt magnets, the general ageing behaviour can be described by an initial sharp loss of the induction corresponding to the short-term irreversible losses discussed above, followed by a much slower reduction leading gradually into a linear decline of induction with time. The initial sharp loss can be avoided by a thermal stabilization process at temperatures slightly higher than operating conditions. For $\mathrm{Sm} \mathrm{CO}_{5}$ magnets, with load line at $-B / \mu_{0} H=1$, the slope of the linear part of the induction losses at temperatures up to $150^{\circ} \mathrm{C}$ is typically $1-2 \times 10^{-7}$. The maximum operating temperatures are approx. $250{ }^{\circ} \mathrm{C}$ for $\mathrm{Sm} \mathrm{Co}, 350{ }^{\circ} \mathrm{C}$ for typical ferrites and $400{ }^{\circ} \mathrm{C}$ for the alnicos.

\section{Development Objectives and Trends}

The common aim of material developers or producers and system designers or users is to maximize performance per unit price, but the criteria of the users as well as the constraints for the producers are so manifold, the field of activity in permanent magnet development will remain wide open for the foreseeable future.

Permanent magnet systems are competing directly with electromagnetic systems, and in many instances they provide a substitute for non-magnetic devices. The three typical directions of development which are pursued today by the materials scientists are high-energy density magnets, magnets with improved mechanical properties such as ductility and, last but not least, low-cost magnets. Remembering the above-mentioned constraints and the great variety of permanent magnet applications, it seems clear that these three development courses are more or less independent from each other.

As to the high-energy density magnets, we come back again to the youngest family, namely the rare earth-cobalt or better rare earth-transition metal alloys $\mathrm{R} T \mathrm{M}$. The primary magnetic properties of all the $\mathrm{R} \mathrm{Co}_{5}$ and $\mathrm{R}_{2} \mathrm{Co}_{17}$ compounds are given in Table 2. Both groups exist as commercially available products whose magnetic properties correspond typically to the curves shown in Fig. 1, but this does not mean that the full potential of these alloys has already been reached. Considerable efforts are still necessary and promise improvement in the demagnetization characteristics. In the $1 / 5$ subgroup, these efforts concentrate on using the optimum alloy $\left(\mathrm{Sm}_{1 \times x y} \operatorname{Pr}_{x} \mathrm{Nd}_{y}\right) \mathrm{Co}_{5}$ seems to head the list - and improving all the technological production parameters such as particle size and shape, powder processing without affecting the alloys which are highly corrosive, perfection of the uniaxial particle alignment and optimization of the heat treatment in order to reach the highest possible coercivity without unreasonably aggravating the problem of magnetizing the material. It is believed that the potential for improvement of the energy product $(\mathrm{BH})_{\max }$ might be around $20 \%$, so that in the very best case, a $\mathrm{RE} \mathrm{Co}_{5}$ permanent magnet with $30 \mathrm{MGOe}$ could be achieved.

The value of 30 MGOe for $(B H)_{\max }$ corresponds to the best results which industry had achieved about five years ago with $R E_{2}$ $\mathrm{TM}_{17}$ - type material. Development here concentrates on improving the coercivity and decreasing its high sensitivity to in creased temperature. This requires better knowledge of the various phase diagrams and of the domain wall behaviour with the dispersed precipitates. Moreover, to bring down the price a considerable part of the cobalt must be replaced by other metals which are cheaper and easier to obtain on the metal market. Before 1978, the year when the cobalt crisis escalated because of the political problems in central Africa, fears for the future price competitiveness of the rare earth-cobalt magnets concerned mainly the high price of samarium. This is a continuing problem as the prices of both rare earth oxides and cobalt might still be subject to important fluctuations and the materials developer must be prepared to meet this situation.

Cobalt price has also had a very severe impact on the alnicos, which are considered to be the classical permanent magnet material for a very broad range of wellestablished technical applications. Today, the alnicos see the advent of a family which is very similar in its magnetic properties, namely the $\mathrm{Fe}-\mathrm{Cr}-\mathrm{Co}$ alloys. At $10-15 \%$ wt., their cobalt content is much lower than that of the alnicos which have between 20 and $35 \%$ wt. A very important feature is their plastic deformability at certain stages of their heat treatment, so that they are suitable for small and intricate shapes. Their magnetic hardness mechanism is basically the same as for alnicos. Complications in the $\mathrm{Fe}-\mathrm{Cr}$-Co alloys in the form of parasitic phases, which impaired both magnetic and mechanical properties, have been overcome by the addition of small amounts of $\mathrm{Nb}, \mathrm{Al}, \mathrm{V}$ or $\mathrm{Ti}$, so that permanent magnets are now commercially available in this alloy.

The field of low-cost magnets is today clearly dominated by the $\mathrm{Ba}$ and Sr ferrites, which are made from cheap raw materials, namely iron oxide and barium or strontium carbonate. Production technology has been subject to a thorough rationalization, so that this material provides the lowest price per unit magnetic energy. The energy products of the sintered magnets are not very far from the theoretical limits, so that only marginal improvements are expected in the future. The drawback of the ferrites
Table 1 -

Mechanisms for Magnetic Hardness

\begin{tabular}{|c|c|c|c|}
\hline \multirow[b]{2}{*}{$\begin{array}{l}\text { Reason for } \\
\text { Magnetic } \\
\text { Hardness }\end{array}$} & Fine Particles & & \multirow[b]{2}{*}{$\begin{array}{l}\text { Mechanical } \\
\text { Hardness }\end{array}$} \\
\hline & $\begin{array}{l}\text { with shape } \\
\text { anisotropy } \\
\text { (precipitation } \\
\text { technology) }\end{array}$ & $\begin{array}{l}\text { with crystal } \\
\text { anisotropy } \\
\text { (powder } \\
\text { technology }\end{array}$ & \\
\hline $\begin{array}{l}\text { Typical } \\
\text { Materials }\end{array}$ & $\begin{array}{l}\text { Alnico } \\
\text { Vicalloy } \\
\text { CuNi Fe }\end{array}$ & $\begin{array}{l}\text { Ba - ferrite } \\
\text { Pt Co } \\
\text { Rare earth } \\
\text { - cobalt }\end{array}$ & $\begin{array}{l}\text { Magnet steels } \\
\text { Remalloy } \\
\text { Mn Al }\end{array}$ \\
\hline
\end{tabular}

(1) Nesbitt E.A. et al., J. App/. Phys. 30 (1959) 365; 32 (1961) 342

(2) Strnat K. et al., J. App/. Phys. 37 (1966) 1252.

(3) Taylor K.N.R., Adv. Phys. 20 (1971) 551.

(4) Menth A. et al., Ann. Rev. Mater. Sci. 8 (1978) 21

$\mathrm{NC}=$ non-collinear spin structure
Table 2 -

Primary Magnetic Properties of $\mathrm{RCo}_{5}$ and $\mathrm{R}_{2} \mathrm{Co}_{17}$ Compounds

\begin{tabular}{|c|c|c|c|c|c|c|}
\hline $\mathrm{R}$ & & $\mathrm{R} \mathrm{Co}_{5}$ & & & $\mathrm{R} \mathrm{Co}_{17}$ & \\
\hline & $4 \pi M_{\mathrm{s}}^{(1)}$ & $T_{\mathrm{C}}^{(3)}$ & $K_{1}^{(4)}$ & $4 \pi M_{\mathrm{s}}^{(2)}$ & $T_{\mathrm{c}}^{(3)}$ & $K_{1}{ }^{(4)}$ \\
$(\mathrm{T})$ & $\left({ }^{\circ} \mathrm{C}\right)$ & $\left(\mathrm{MJ} / \mathrm{m}^{3}\right)$ & $(\mathrm{T})$ & $\left({ }^{\circ} \mathrm{C}\right)$ & $\left(\mathrm{MJ} / \mathrm{m}^{3}\right)$ \\
$\mathrm{Ce}$ & 0.85 & 374 & 5.3 & 1.15 & 800 & -0.6 \\
$\mathrm{Pr}$ & 1.12 & 612 & 8.1 & 1.38 & 890 & -0.6 \\
$\mathrm{Nd}$ & 1.20 & 630 & 0.7 & 1.39 & 900 & -1.1 \\
$\mathrm{Sm}$ & 0.97 & 724 & 17.2 & 1.20 & 920 & 3.3 \\
$\mathrm{Gd}$ & 0.19 & 735 & 4.6 & 0.73 & 930 & -0.5 \\
$\mathrm{~Tb}$ & 0.24 & 707 & $\mathrm{NC}$ & 0.68 & 920 & -3.3 \\
$\mathrm{Dy}$ & 0.30 & 693 & $\mathrm{NC}$ & 0.70 & 910 & -2.6 \\
$\mathrm{Ho}$ & 0.53 & 727 & 3.6 & 0.83 & 920 & -1.0 \\
$\mathrm{Er}$ & 0.63 & 713 & 3.8 & 0.90 & 930 & 0.41 \\
$\mathrm{Tm}$ & 0.67 & 747 & - & 1.13 & 920 & 0.50 \\
$\mathrm{Yb}$ & - & - & - & - & - & -0.38 \\
$\mathrm{Lu}$ & - & - & - & 1.27 & 940 & -0.20 \\
$\mathrm{La}$ & 0.91 & 567 & 5.9 & - & - & - \\
$\mathrm{Y}$ & 1.06 & 648 & 5.2 & 1.25 & 940 & -0.34 \\
$\mathrm{Th}$ & - & - & 2.6 & - & - & -0.53 \\
\hline
\end{tabular}


is their unsatisfactory temperature stability and their relatively low flux density.

The material which has the potential to replace the ferrites in many applications is the $\mathrm{Mn}-\mathrm{Al}-\mathrm{C}$ alloy which was discovered as a permanent magnet material around 1958. The saturation magnetization and the temperature coefficients are more favourable than with the ferrites. It has been shown that the domain walls in this material are related to antiphase boundaries and crystal defects of various kinds. The $\tau$-phase, which is not stable with temperature, is stabilized to some extent by the carbon addition. Unfortunately, this $\tau$-phase does not grow uniaxially in the material and it is only by mechanical orientation through hot extrusion that some alignment of the magnetically easy axes can be achieved. In small quantities, this very promising material with energy product $(B H)_{\text {max }} \cong 5.5$ MGOe is available on the market. It seems that further development of the material or the extrusion process will be necessary.

\section{BIBLIOGRAPHY}

- Cullity B.D Introduction to Magnetic Materials (Addison - Wesley) 1972

Schüler K. and Brinkmann K., Dauermagnete (Springer) 1970.

Zijlstra H., "Trends in Permanent Magnet Material Development" I.E.E.E. Trans. Magn., MAG-14 (Sept. 1978) 5, pp. 661-664.

Weinmann D., "Recoma-New Standards in Permanent Magnet Applications" Goldschmidt informeirt 2/79 48, pp. 30-42.

- Livingston J.D., "Microstructure and Coerci vity of Permanent Magnet Materials" General Electric - Technical Information Series, Report No. 80, CRD 139, July 1980.

\title{
Mixed Valence Compounds of the Rare Earths
}

\section{A Fascinating Class of Solids}

\author{
E. Müller-Hartmann, Cologne \\ (Institut für Theoretische Physik, Universität zu Köln)
}

The discovery of extraordinary substances or of new classes of substances with extraordinary properties has always added to the liveliness and relevance of solid state physics. One such class of unusual substances is certainly the mixed valence lanthanide compounds. Based upon them an important new branch of research in the field of magnetism has developed over the past decade which contains interesting implications for the understanding of condensed matter in general.

For some time it has been known that various rare earth compounds have lattice spacings with anomalous values. Since the ionic radii of the rare earths vary noticeably with their valence, it was obvious to chemists that something unusual had happened to the valence of the rare earths in these compounds. However, it was not before the late sixties that physicists fully appreciated that these compounds were exotic solids

The characteristic common feature of mixed valence compounds is believed to be that their rare earth constituents are not in a stable state of well defined valence, but coexist in two different valence states corresponding to two distinct atomic configurations of the $4 f$-shells, e.g. $4 f^{n}$ and $4 f^{n-1}$. It is furthermore believed that the two configurations are not associated with different lattice sites - this would be called an inhomogeneously mixed valence - but that both of them are found on each rare earth atom in the compound: the valence is homogeneously mixed.
In the Table overleaf, a representative list of mixed valence compounds is given; some thirty known compounds appear to have mixed valence at normal pressure, others go into a mixed valence state at high pressure. Not included in the Table are the numerous mixed valence alloys and rare earth impurities with mixed valence in various environments.

Evidence for the coexistence of two dif ferent $4 \mathrm{f}$-configurations is derived through several experimental methods. The most direct evidence probably comes from X-ray photoelectron spectroscopy (XPS) where the spectrum of the photoelectrons directly reflects the excitation spectrum of the $4 f$-configurations left behind, i.e., $4 f^{n-1}$ and $4 f^{n-2}$. Unfortunately, such evidence cannot be obtained for all compounds in question. In particular, XPS analysis does not apply to Ce metal and its compounds, as it is impossible to draw photoelectrons out of the configuration $4 f^{0}$. This leads to some uncertainty whether all the compounds listed in the Table have two coexisting $4 f$-configurations. However, there is overwhelming evidence that the majority of compounds listed contain two configurations of their rare earth $4 \mathrm{f}$-shells.

A second body of evidence is needed to support the assumption of homogeneous valence mixing. Various properties of the compounds point in this direction. Again, there is a method which is particularly well suited to distinguish between inhomogeneous and homogeneous mixing: Mössbauer spectroscopy identifies 4f-configurations, as the chemical shift varies with the

\section{Deep Levels in} Semiconductors

\section{Jaros}

An authoritative survey of the physics of deep levels in semiconductor materials and their influence on the functioning of semiconductor devices. It summarises recent research efforts and provides both a conceptual framework and a clear prescription for dealing with problems concerning the theory, modelling, detection and identification of deep levels.

May $1982 \mathrm{xi}+302 \mathrm{pp}$ 0-85274-516-8 £24.00

\section{Weak Interactions}

\section{Second edition}

\section{Bailin}

Graduate Student Series in Physics

A revised edition of this successful graduate text, now extended to include the dramatic experimental results of recent years and to discuss the standard unified field theory of weak and electromagnetic interactions. The author develops the fundamenta phenomenology of weak interactions within the field theory framework which has become indispensable following the success of the unified gauge theory.

$$
\text { July } 1982 x i+458 p p
$$$$
\text { 0-85274-539-7 £11.95 }
$$

\section{Instrument Science and Technology Volume 1}

\section{B E Jones (ed)}

The first volume of an important new series on measurement and instrumentation. The 13 chapters cover noise and measurement mathematical modelling, parameter estimation methods, dynamics, systematic design, feedback, reliability and ergonomics. The book will be useful to all instrument designers and metrologists, and to those teaching the subject.

January $1982 x+144 p p$

0-85274-438-2 f6.95

\section{Adam Hilger Ltd}

Techno House

Redcliffe Way

Bristol BS1 6NX

England 